\title{
First in Man Topical Treatment of Melanoma with Solasodine Glycosides in a Formulation Curaderm: A Case Report
}

\author{
B. E. Cham \\ Australasian Medical Research, Port Vila, Republic of Vanuatu \\ Email: bill.cham@gmail.com
}

How to cite this paper: Cham, B.E. (2020) First in Man Topical Treatment of Melanoma with Solasodine Glycosides in a Formulation Curaderm: A Case Report. Journal of Cancer Therapy, 11, 617-630. https://doi.org/10.4236/jct.2020.1110052

Received: September 18, 2020

Accepted: October 19, 2020

Published: October 22, 2020

Copyright $\odot 2020$ by author(s) and Scientific Research Publishing Inc. This work is licensed under the Creative Commons Attribution International License (CC BY 4.0).

http://creativecommons.org/licenses/by/4.0/

\section{(c) (i) Open Access}

\begin{abstract}
Background: There is ample evidence to support the safety and efficacy of the topical anticancer cream Curaderm in the treatment of non-melanoma skin cancers. Curaderm contains the natural glycoalkaloid solamargine in the form of BEC, which has been established as a novel antineoplastic agent. BEC is the initials of the inventor of the described technology. It is known that BEC expresses anti-melanoma properties in cell culture and animals. Because of potential metastasis, clinical work with BEC on melanoma was stalled. However, recent studies show that BEC has anti-metastatic properties and this, together with currently better understanding of the mode of anti-cancer actions of BEC, has led to the treatment of a patient who refused to have surgery for her clinically diagnosed stage II melanoma. Treatment: A 67-year woman had a birthmark that developed into a clinically diagnosed stage II melanoma and was treated with topical application of Curaderm twice daily for 7 weeks. Results: The pattern of response of the melanoma to Curaderm therapy was similar to that observed when basal cell carcinoma is treated with Curaderm. The melanoma responded rapidly to the treatment and in 7 weeks the lesion was removed with no demonstrable side effects. The cosmetic end result was very acceptable. Conclusion: The clinical resolution of the melanoma with Curaderm pharmacotherapy conforms to the cell culture and animal observations that solasodine rhamnosides, and thus Curaderm, is very specific and efficacious for the first in man treatment of melanoma, creating the possibility of a simple treatment for melanoma. Further investigations with controlled clinical trials are warranted.
\end{abstract}

\section{Keywords}

Melanoma, Curaderm, BEC, Solamargine, Non-Melanoma Skin Cancer, Metastasis 


\section{Introduction}

Melanoma is a serious form of skin cancer that starts in cells known as melanocytes.

Melanoma is more dangerous than other skin cancers because of its ability to spread to other organs more rapidly.

Approximately 132,000 new cases of melanoma are diagnosed worldwide each year, according to the World Health Organization.

Melanoma is the least common but the most deadly skin cancer, accounting for only about $1 \%$ of all cases, but the vast majority of skin cancer death. In 2020, it is estimated that there will be 100,350 new cases of melanoma in the United States and 6850 deaths from the disease [1].

Melanoma of the skin is the third most commonly diagnosed cancer in Australia (excluding non-melanoma skin cancer). In 2015, 13,694 new cases were diagnosed in Australia, and in 2018, 1429 people died [2].

Annual treatment costs for melanoma ranged from US\$44.9 million among Medicare patients with existing cases to US\$932.5 million among newly diagnosed cases across all age groups in the USA [3].

Australia is considered to be the skin cancer capital of the world, and, in Australia, the lifetime cost of skin cancer is estimated at $\$ 44,796$ per melanoma and $\$ 2459$ per non-melanoma skin cancer [4].

There are different types of melanomas and there are unique differences among each type. Cutaneous melanoma, or melanoma of the skin, is the most common type of melanoma worldwide. Five stages of melanomas have been defined ranging from Stage 0 to Stage IV with Stage IV being the most serious.

This communication centres on a Stage II melanoma case. Stage II melanoma is usually treated by surgery (wide excision) to remove the melanoma and a margin of normal skin around it. Although not popular with all doctors, imiquimod cream or radiation therapies are also used instead of surgery.

In 1987 it was first reported that plant-derived glycoalkaloids solasodine glycosides, including solamargine, solasonine, mono- and di-glycosides of solasodine, known as BEC [5], induced remarkable antineoplastic effects in cell culture [6] and in animals [7] and anticancer effects in humans [8] [9] [10] [11] [12].

Since then, a plethora of further investigations has resulted in the placement of BEC and its individual components as very promising antineoplastic agents with vast potential to serve as targeted anticancer agents [13]. With BEC, solamargine accounts for $86 \%$ antineoplastic activity and solasonine accounts for $9 \%$ antineoplastic activity, whereas, the mono- and di-glycosides of solasodine contribute $5 \%$ anticancer activity. The anticancer activity of these glycoalkaloids is considered to be concerted and additive [14].

The governing principle that determines the potency of antineoplastic activity is the plant sugar rhamnose that forms part of the glycoalkaloids [15]. BEC targets specific mutant proteins on cancer cell membranes that act as specific receptors. After binding to these characterised specific receptors [16] [17] [18] [19] [20], BEC is internalised by cell receptor-mediated endocytosis followed by the 
anticancer sequelae of identifiable antineoplastic properties on a variety of biological pathways, including cell survival pathways [21], tumour suppressor pathways [22], lysosomal pathways [23], mitochondrial pathways [24], caspase activation pathways [25], death receptor pathways [26], protein kinase pathways [27] and signal pathways that impede invasion/migration [16] [27] and multidrug resistance [16] [28].

BEC exhibits much higher cytotoxic effects on cancer cells than currently used antineoplastic agents such as vinblastine, vincristine, camptothecin, cisplatin, 5-fluorouracil, gemcitabine, epirubicin, cyclophosphamide, taxol and doxorubicin [29].

Furthermore, the absolute concentrations of these drugs to obtain comparable efficacy as BEC is in the order of 6 - 40 times higher [30].

Moreover, the therapeutic index (TI: also referred to as the therapeutic ratio) is much higher for BEC compared with other antineoplastic agents as shown with cell culture studies [31] and animal studies [32]. The high TI of BEC translates to high safety margins.

BEC is active against a wide variety of cancer cells, such as ovarian cancer [33]), basal cell carcinoma [34], squamous cell carcinoma [35], melanoma [36], colorectal cancer [37], bladder cancer [38], oral epidermoid carcinoma [39], breast cancer [28], leukemia [40], prostate cancer [41], liver cancer [42], lung cancer [43], pancreatic cancer [44], gastric carcinoma [45], renal cancer [34], uterine cancer [33], mesothelioma [46], glioblastoma [47] and osteosarcoma [22].

In addition, BEC has curative properties in animals with terminal cancer [7] [15].

Earlier cell culture studies with melanoma showed that BEC, in a dose dependent manner, caused apoptosis and was very effective against melanoma cells with a high safety margin $(\mathrm{TI}=3.6)$ [30]. It was also reported that intra-lesion injection of BEC into a large melanoma on a horse resulted in elimination of the melanoma [31].

Later studies with Solanum incanum extract, that is similar to BEC, confirmed that the extract induced apoptosis in murine B16 melanoma cells in vitro.

Intralesion injection in vivo decreased the tumour size of the regional melanoma in the footpad. Moreover, intraperitoneal injection of the extract inhibited the growth and the number of established melanoma metastases in the lungs [36].

The specificity of BEC towards cancer cells has been confirmed by many investigators. In particular, a recent study has reported the effect of solamargine (the main active anticancer component in BEC) on human melanoma cancer cells [23].

The investigators demonstrated that solamargine rapidly, selectively and effectively inhibited the growth of metastatic and primary melanoma cells, with minimum effect on normal and benign melanoma cells. Solamargine showed high efficacy in vitro, particularly against the vertical growth phase of melanoma 
cells.

Recent studies have demonstrated that solamargine has potent anti-metastatic properties [27] [36] [44].

BEC, in a pharmacotherapy cream formulation Curaderm, has been approved in Europe as a Medical Device Class 1 for the indication of topical treatment for basal cell carcinoma of the skin. Studies and publications attest to the efficacy and safety of Curaderm in the treatment of nonmelanoma skin cancers resulting in remarkable cosmetic outcomes [8] [9] [10] [11] [12] [48]-[62].

The contra-indication for Curaderm to date has been, to avoid treatment of melanoma, because it was considered that melanoma could possibly metastasise during Curaderm therapy.

However, more recent studies have reported that BEC and/or its components display anti-metastatic properties by blocking epithelial mesenchymal transition [63], and suppressing the invasive capacity of cancer cells by downregulation of Matrix metalloproteinases (MMPs) MMP-2 and MMP-9 expressions and activities [27].

With this information, it seemed reasonable to test Curaderm pharmacotherapy on a clinically diagnosed cutaneous stage II melanoma, especially because the patient refused surgical or other procedures.

\section{Patient}

A 67-year female subject had a birthmark on the left upper arm that remained unremarkable up until 4 months prior to treatment. During those 4 months, the patient observed that the birthmark started to change by rising above the skin and becoming very itchy. The surface of the lesion had changed by the appearance of multiple circles inside the lesion. Ulceration started by the appearance of a tiny opening within the lesion, which secreted a clear fluid that avidly attracted flies. The border of the lesion started to change and was becoming asymmetrical. At the time of examination, the lesion was approximately $2.3 \mathrm{~cm}$ wide and protruded approximately $6 \mathrm{~mm}$ from the skin. Prior to the observed changes, there was no protrusion and the lesion was flat. The patient did not suffer from other conditions and appeared healthy. Physical examination revealed that there were no regional palpable and axillary lymph node involvements. The patient was scalpel-phobic and refused to have a biopsy taken. Without a biopsy, it was not possible to accurately measure the Breslow thickness. The following changes were observed over the 4 months prior to Curaderm pharmacotherapy. The lesion became asymmetrical, the border of the lesion became irregular, there was swelling within the lesion and the overall changes followed an evolutionary pattern. According to the American Academy of Dermatology descriptions, the lesion was classified as a stage II melanoma [64].

Curaderm is a cream formulation for topical use for actinic keratosis and non-melanoma skin cancer. Curaderm contains $5 \mathrm{mg} \%$ BEC, $5 \%$ urea and $10 \%$ salicylic acid in a stabilised cetomacrogol base. Curaderm was supplied by Curaderm Global Limited. 


\section{Treatment with Curaderm}

Just prior to the commencement with Curaderm therapy, 50 micrograms of BEC in 50 microliters of Dimethylsulphoxide (DMSO) was topically applied to the lesion and beyond the lesion to test for possible sensitivity [65].

The following treatment procedure of Curaderm was explained to the patient:

1) Wash the lesion and the surrounding area with a mild non-irritating soap.

2) Rinse with water.

3) Dry thoroughly.

4) Unscrew the lid of the Curaderm tube and remove the protective foil that covers the aperture in the lid of the tube.

5) Apply Curaderm cream to the lesion by gently squeezing the tube, just enough cream to cover the lesion. Do not apply the cream in large quantity and do not extend the cream more than $0.5 \mathrm{~cm}$ onto the apparently normal skin surrounding the edge of the lesion.

6) Cover the lesion with an occlusive dressing (micropore) until the next application of Curaderm.

7) Apply the cream to the lesion twice daily every 12 hours.

8) Stop treatment only when the lesion has been completely ablated and replaced with normal skin.

\section{Results}

Figure 1 illustrates the lesion before treatment with Curaderm. The diameter of the melanoma was $2.3 \mathrm{~cm}$ (Figure 1(a)) and protruded $6 \mathrm{~mm}$ from the skin surface with slight swelling of the lesion that contained some hair follicles (Figure $1(\mathrm{~b}))$.

Topical application of BEC in DMSO solution showed no sensitivity effect. With Curaderm pharmacotherapy the melanoma responded rapidly. Swelling and erythema of the lesion occurred after the second application of Curaderm. This was followed by ulceration of the lesion with deeper tissue involvement. During ulceration, the lesion appeared fragmented and, thereafter, new apparently normal cell growth ensued.

Figure 2 shows the lesion at 4 weeks of treatment with Curaderm. Treatment continued until there was no further reaction of Curaderm with the lesion. The total treatment period was 7 weeks.

After treatment, the lesion appeared completely clean, devoid of any fragmented particles with some scar tissue in the centre of where the melanoma was.

Figure 3(a), Figure 3(b) illustrate the lesion 7 weeks after commencement of treatment. A clear demarcated area where the lesion was is observable. Although Curaderm was applied beyond the melanoma (approximately $0.5 \mathrm{~cm}$ surrounding the melanoma), no reaction with the normal skin was observed and a slight indentation of the skin where the lesion was prior to the treatment was seen (Figure 3(b)). The patient did not experience any pain at the site of Curaderm application or elsewhere. 


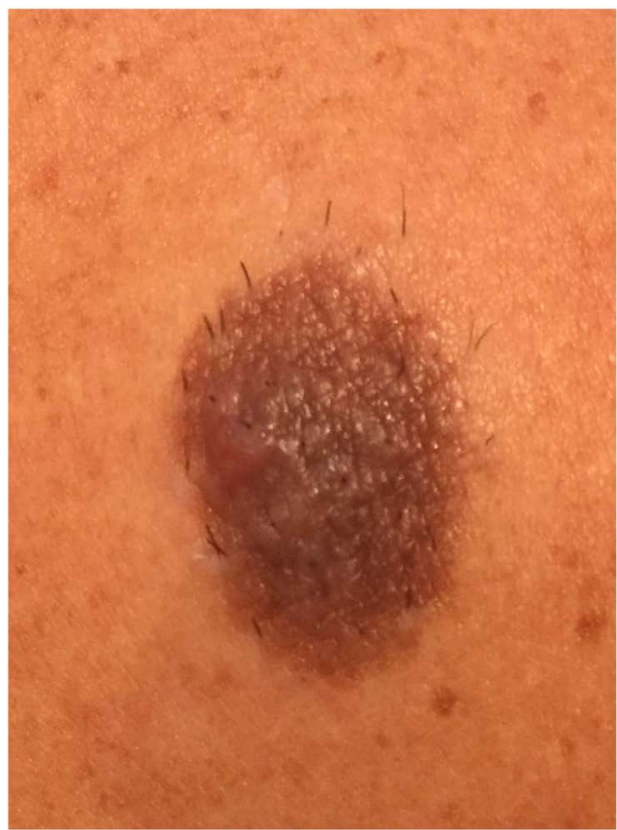

(a)

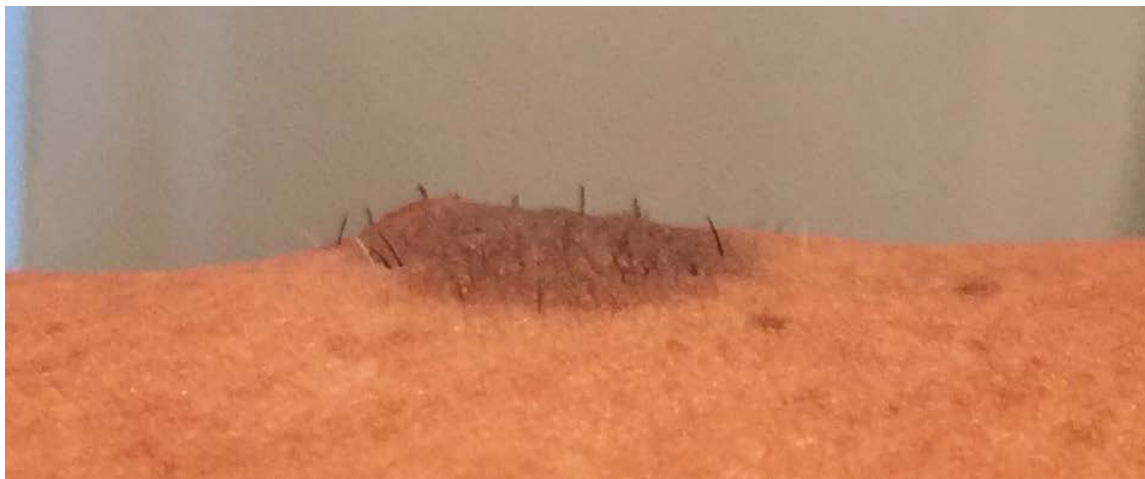

(b)

Figure 1. (a) Before treatment the lesion was approximately $2.3 \mathrm{~cm}$ (actual scale 1:1) in diameter and had changed significantly during the previous 4 months; (b) Before treatment the lesion protruded approximately $6 \mathrm{~mm}$.

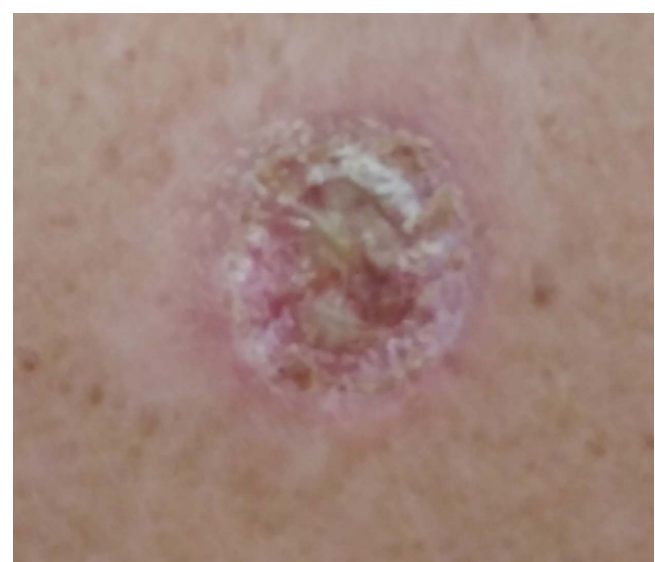

Figure 2. At 4 weeks of treatment the lesion eroded deep down to the dermal layer. 


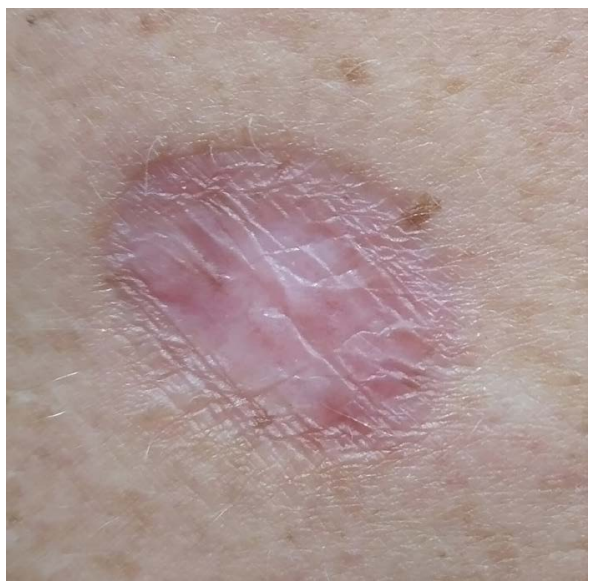

(a)

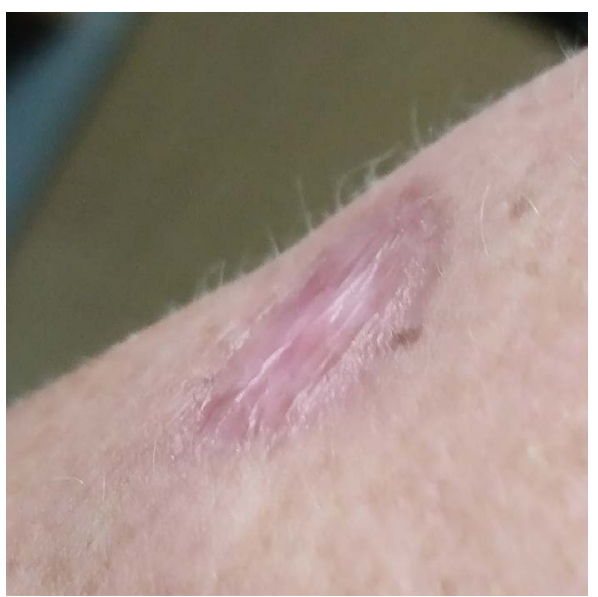

(b)

Figure 3. (a) The lesion 7 weeks after commencement of treatment with Curaderm showing a clear demarcated area where the lesion was; (b) No reaction with the normal skin was observed and a slight indentation of the skin is observed where the lesion was prior to the treatment.

\section{Discussion}

The incidence of malignant melanoma and consequential mortality is on the rise. Malignant melanoma has the highest mortality rate of all skin cancers. Surgical excision represents the primary treatment for malignant melanoma. On occasions, however, for a variety of reasons, surgery may not be possible, or refused by the patient's personal wishes.

Topical Curaderm is a cream formulation that contains BEC. BEC is composed of a mixture of naturally occurring glycoalkaloids of which solamargine and solasonine are the main components. Curaderm has been approved as a Class I Medical Device in Europe for the treatment of basal cell carcinoma. In recent years Curaderm has been shown to also be effective against keratosis and in situ squamous cell carcinoma.

BEC is a promising antineoplastic agent for melanoma as shown in cell culture studies and animal studies [30] [31] [36]. However, because of possible me- 
tastasis, and thus potentially serious ramifications, not much further clinical work with BEC and melanoma has transpired.

Recent studies have shown that BEC has anti-metastatic properties and this, together with better understanding of the antineoplastic mechanism of action of BEC, has opened possibilities for the application of BEC to metastasis-prone cancers.

Such reported studies have led to the treatment of a stage II clinically diagnosed malignant melanoma with Curaderm, resulting in the elimination of the lesion with no demonstrable side effects. A shortcoming of the current study is, that due to the patient's insistence, no biopsies were taken before and after treatments. However, it has been shown that solamargine triggers cellular necrosis rapidly, selectively and effectively in metastatic and primary melanoma cells, with minimum effect on normal and benign melanoma cells [23]. Such observations give credence that the clinically diagnosed melanoma treated with Curaderm was malignant.

The pattern of response of the melanoma to Curaderm therapy was similar to those observed when cutaneous basal cell carcinomas are treated with Curaderm [9].

Compelling evidence of safety has previously been reported when patients applied Curaderm twice daily for 14 weeks [8] [9] [16] [31] [60].

The cosmetic end result was very acceptable, the patient is being followed up. To date the patient has been followed up for over six months after cessation of Curaderm pharmacotherapy and there has been no relapse of the lesion. It is interesting that when Curaderm was applied to the surrounding apparently normal skin, no reactions were observed, in accordance with the treatment findings of non-melanoma skin cancers with Curaderm therapy [8] [9] [16] [30] [60] [65]. This indicates specificity and selectivity of Curaderm towards cancer cells, as has been shown in cell culture and animal studies.

During treatment with Curaderm, an indentation occurred at the area where the melanoma was positioned. The depth of the indentation had progressed into the dermal layer of the skin, providing some support that the lesion was a stage II melanoma. During the latter stage of the treatment, the lesion stopped ulcerating and then reverse coursed and slowly healed. Minor complications have been reported with Curaderm pharmacotherapy when applied to basal cell carcinoma and squamous cell carcinoma in situ. In such cases, a small number of patients have experienced transient pain, pruritis, and irritation at the application site of Curaderm cream [8] [9] [20] [50] [52] [53] [66]. However, in the current case with the melanoma patient, no pain at the application site or elsewhere was experienced.

This result was obtained from a single clinically diagnosed stage II melanoma case. As with all case reports thus far, long follow-up to definitely confirm that this therapy may be effective in treating melanoma in situ is essential. Further investigations with controlled clinical trials are warranted and necessary. Phase 
III trials with Curaderm for the indication of stage 0, in situ, melanoma are planned.

\section{Conclusion}

Curaderm, containing solamargine and solasonine in the form of BEC, is very effective and safe in the treatment of actinic keratosis, basal cell carcinoma and in situ squamous cell carcinoma. Cell culture and animal studies with BEC have resulted in the safe elimination of a wide variety of cancers, including melanoma. The fact that BEC additionally has anti-metastatic properties has led to the first in man treatment of melanoma with Curaderm. Seven weeks of Curaderm therapy resulted in the removal of the melanoma with a good cosmetic outcome. Many studies are necessary to determine the safety and efficacy of BEC and Curaderm for the possible treatment of melanoma. Until then, surgical procedures for melanoma treatments are recommended.

\section{Statement of Ethics}

Informed consent was obtained from the patient who refused to have surgery or other treatment regimens.

\section{Conflicts of Interest}

The author declares no conflicts of interest regarding the publication of this paper.

\section{References}

[1] American Cancer Society (2020) Cancer Facts and Figures 2020. American Cancer Society, Atlanta.

[2] Cancer Council Australia Skin Cancer Statistics \& Issues (2020) Skin Cancer Incidence and Mortality.

[3] Guy, G.P., Ekwueme, D.U., Tangka, F.K. and Richardson, L.C. (2012) Melanoma Treatment Costs. A Systematic Review of the Literature, 1990-2011. American Journal of Preventive Medicine, 43, 537-545. https://doi.org/10.1016/j.amepre.2012.07.031

[4] Doran, C.M., et al. (2015) Estimating the Economic Costs of Skin Cancer in New South Wales, Australia. BMC Public Health, 15, Article No. 952. https://doi.org/10.1186/s12889-015-2267-3

[5] Cham, B.E. and Wilson, L. (1987) HPLC of Glycoalkaloids from Solanum sodomaeum. Planta Medica, 1, 59-62. https://doi.org/10.1055/s-2006-962621

[6] Daunter, B. and Cham, B.E. (1990) Solasodine Glycosides. In Vitro Preferential Cytotoxicity for Human Cancer Cells. Cancer Letters, 55, 209-220.

https://doi.org/10.1016/0304-3835(90)90121-D

[7] Cham, B.E., Gilliver, M. and Wilson, L. (1987) Antitumour Effects of Glycoalkaloids Isolated from Solanum sodomaeum. Planta Medica, 1, 34-36. https://doi.org/10.1055/s-2006-962612

[8] Cham, B.E. and Meares, H.M. (1987) Glycoalkaloids from Solanum sodomaeum L. Are Effective in the Treatment of Skin Cancers in Man. Cancer Letters, 36, 111-118. 
https://doi.org/10.1016/0304-3835(87)90081-4

[9] Cham, B.E., Daunter, B. and Evans, R. (1991) Topical Treatment of Malignant and Premalignant Skin Cancers by Very Low Concentrations of a Standard Mixture of Solasodine Glycosides. Cancer Letters, 59, 183-192.

https://doi.org/10.1016/0304-3835(91)90140-D

[10] Punjabi, S., Cook, I., Kersey, P., Marks, R., Finlay, A., Sharpe, G. and Cerio, R. (2000) A Double Blind, Multi-Centre Parallel Group Study of BEC-5 Cream in Basal Cell Carcinoma. The Journal of the European Academy of Dermatology and Venereology, 14, 47-60.

[11] Batsev, A.F., Dobrokhotova, V.Z. and Cham, B.E. (2016) Topical Cream Curaderm ${ }^{\text {bec5 }}$ Treats a Recalcitrant Basal Cell Carcinoma. Clinical Medical Reviews and Case Reports, 3, 098. https://doi.org/10.23937/2378-3656/1410098

[12] Goldberg, L.H., Landau, J.M., Moody, M.N. and Vergilis-Kalner, I.J. (2011) Treatment of Bowen's Disease on the Penis with Low Concentrations of a Standard Mixture of Solasodine Glycosides and Liquid Nitrogen. Dermatologic Surgery, 37, 858-861. https://doi.org/10.1111/j.1524-4725.2011.02014.x

[13] Cham, B.E. (2020) Combination Treatment with BEC and Cisplatin Synergistically Augments Anticancer Activity and Results in Increased Absolute Survival. Journal of Cancer Therapy, 11, 470-482. https://doi.org/10.4236/jct.2020.118040

[14] Cham, A., Cham, K., Chase, T. and Cham, B. (2015) A Standardized Plant Extract Containing a Target Compound Is Acceptable as a Potent Therapeutic Entity: Relevance to BEC and Solamargine, a Topical Clinical Formulation Curaderm ${ }^{\text {BEC5. }}$. Journal of Cancer Treatment and Research, 3, 22-27. https://doi.org/10.11648/j.jctr.20150302.12

[15] Cham, B.E. and Daunter, B. (1990) Solasodine Glycosides. Selective Cytotoxicity for Cancer Cells and Inhibition of Cytotoxicity by Rhamnose in Mice with Sarcoma 180. Cancer Letters, 55, 221-225. https://doi.org/10.1016/0304-3835(90)90122-E

[16] Cham, B.E. (2017) Solasodine, Solamargine and Mixtures of Solasodine Rhamnosides: Pathway to Expansive Clinical Anticancer Therapies. International Journal of Clinical Medicine, 8, 692-713. https://doi.org/10.4236/ijcm.2017.812064

[17] Lipscombe, R., Carter, S. and Ruane, M. (2005) Rhamnose Binding Protein. USA Patent No. 6930171.

[18] Cham, B.E. (2007) Solasodine Rhamnosyl Glycosides Specifically Bind Cancer Cell Receptors and Induce Apoptosis and Necrosis. Treatment for Skin Cancer and Hope for Internal Cancers. Research Journal of Biological Sciences, 2, 503-514.

[19] Wang, Y., Gao, J., Gu, G., Li, G., Cui, C., Sun, B., et al. (2011) In Situ RBL Receptor Visualisation and Its Mediated Anticancer Activity for Solasodine Rhamnosides. ChemBioChem, 12, 2418-2420. https://doi.org/10.1002/cbic.201100551

[20] Cham, B.E. (1994) Solasodine Glycosides as Anticancer Agents: Preclinical and Clinical Studies. Asia Pacific Journal of Pharmacology, 9, 113-118.

[21] An, W., Lai, H., Zhang, Y., Liu, M., Lin, X. and Cao, S. (2019) Apoptotic Pathway as the Therapeutic Target for Anticancer Traditional Chinese Medicines. Frontiers in Pharmacology. https://doi.org/10.3389/fphar.2019.00758

[22] Li, X., Zhao, Y., Wu, W.K., Liu, S., Cui, M. and Lou, H. (2011) Solamargine Induces Apoptosis with p53 Transcription-Dependent and Transcription-Independent Pathways in Human Osteosarcoma U2OS Cells. Life Sciences, 88, 314-321. https://doi.org/10.1016/j.lfs.2010.12.006

[23] Al Sinani, S.S., Eltayeb, E.A., Coomber, B.L. and Adham, S.A. (2016) Solamargine 
Triggers Cellular Necrosis Selectively in Different Types of Human Melanoma Cancer Cells through Extrinsic Lysosomal Mitochondrial Death Pathways. Cancer Cell International, 16, 287-294. https://doi.org/10.1186/s12935-016-0287-4

[24] Zang, X., Yan, Z., Xu, T., An, Z., Chen, W., Wang, X., Huang, M. and Zhu, F. (2018) SM Derived from Solanum nigrum Induces Apoptosis of Human Cholangio Carcinoma QBC939 Cells. Oncology Letters, 15, 6329-6335.

https://doi.org/10.3892/ol.2018.8171

[25] Al Sinani, S.S.S. and Eltayeb, E.A. (2017) The Steroidal Glycoalkaloids Solamargine and Solasonine in Solanum Plants. South African Journal of Botany, 112, 253-269. https://doi.org/10.1016/j.sajb.2017.06.002

[26] Kalalinia, F. and Korimi-Sani, I. (2017) Anticancer Properties of Solamargine: A Systematic Review. Phytotherapy Research, 31, 858-870. https://doi.org/10.1002/ptr.5809

[27] Sani, I.K., Marashi, S.H. and Kalalinia, F. (2015) Solamargine Inhibits Migration and Invasion of Human Hepatocellular Carcinoma Cells through Down-Regulation of Matrix Metalloproteinases 2 and 9 Expression and Activity. Toxicology in Vitro, 29, 893-900. https://doi.org/10.1016/j.tiv.2015.03.012

[28] Li, X., Zhao, Y., Liu, S., Cui, M. and Lou, H. (2011) Induction of Actin Disruption and Downregulation of P-Glycoprotein Expression of Solamargine in Multidrug-Resistant K562/A02 Cells. Chinese Medical Journal, 124, 2038-2044.

[29] Cham, B.E. (2013) Drug Therapy: Solamargine and Other Solasodine Rhamnosyl Glycosides as Anticancer Agents. Modern Chemotherapy, 2, 33-49.

https://doi.org/10.4236/mc.2013.22005

[30] Cham, B.E. (2013) Inspired by Nature, Proven by Science. The New Generation Cancer Treatment That Causes Cancer Cells to Commit Suicide. Colorite Graphics, Book, 264 p.

[31] Cham, B.E. (2007) Solasodine Rhamnosyl Glycosides Specifically Bind Cancer Cell Receptors and Induce Apoptosis and Necrosis. Treatment for Skin Cancer and Hope for Internal Cancers. Research Journal of Biological Sciences, 2, 503-514.

[32] Cham, B.E. and Daunter, B. (1990) Solasodine Glycosides. In Vitro Preferential Cytotoxicity for Human Cancer Cells. Cancer Letters, 55, 209-220.

https://doi.org/10.1016/0304-3835(90)90121-D

[33] Wu, Y.H., Chiu, W.T., Young, M.J., Chang, T.H., Huang, Y.F. and Chou, C.Y. (2015) Solanum Incanum Extract Down Regulates Aldehyde Dehydrogenase 1-Medicated Stemness and Inhibits Tumor Formation in Ovarian Cancer Cells. Journal of Cancer, 6, 1011-1019. https://doi.org/10.7150/jca.12738

[34] Cham, B.E. and Daunter, B. (1990) Topical Treatment of Pre-Malignant and Malignant Skin Cancers with Curaderm. Drugs of Today, 26, 55-58.

[35] Wu, C.H., Liang, C.H., Shiu, L.Y., Chang, L.C., Lin, T.S., Lan, C.C.E., Tsai, J.C., Wong, T.W., Wei, K.J., Lin, T.X., Chang, N.S. and Sheu, H.M. (2011) Solanum incanum Extract (SR-T100) Induces Cutaneous Squamous Cell Carcinoma Apoptosis through Modulating Tumour Necrosis Factor Receptor Signalling Pathway. Journal Dermatological Science, 63, 83-92. https://doi.org/10.1016/j.jdermsci.2011.04.003

[36] Yu, S., Sheu, H.M. and Lee, C.H. (2017) Solanum Incanum Extract (SR-T100) Induces Melanoma Cell Apoptosis and Inhibits Established Lung Metastasis. Oncotarget, 8, 103509-103517. https://doi.org/10.18632/oncotarget.21508

[37] Wu, J., Tang, X., Ma, C., Shi, Y., Wu, W. and Hann, S. (2020) The Regulation and Interaction of Colon Cancer-Associated Transcript-1 and miR7-5p Contribute to the Inhibition of SP1 Expression by Solamargine in Human Nasopharyngeal Carci- 
noma Cells. Phytotherapy Research, 34, 201-213. https://doi.org/10.1002/ptr.6555

[38] Ning, P.H. (2016) Evaluation of Solamargine as a Therapeutic in Bladder Cancer. Scholar Bank@NUS Repository.

[39] Cham, B.E. (2008) Cancer Intralesion Chemotherapy with Solasodine Rhamnosyl Glycosides. Research Journal of Biological Sciences, 3, 1008-1017.

[40] Sun, L., Zhao, Y., Li, X., Yuan, H., Cheng, A. and Lou, H. (2010) A Lysosomal-Mitochondrial Death Pathway Is Induced by Solamargine in Human K562 Leukemia Cells. Toxicology in Vitro, 24, 1504-1511. https://doi.org/10.1016/j.tiv.2010.07.013

[41] Xiang, S.T., Zhang, Q.H., Tang, Q., Zheng, F., Wu, J.J., Yang, L.J. and Hann, S.S. (2016) Activation of AMPKalpha Mediates Additive Effects of Solamargine and Metformin on Suppressing MUC 1 Expression in Castration-Resistant Prostate Cancer Cells. Scientific Reports, 6, Article No. 36721. https://doi.org/10.1038/srep36721

[42] Kuo, K.W., Hsu, S.H., Li, Y.P., Lin, W.L., Liu, L.F., Chang, L.C., Lin, C.N., Lin, C.C. and Sheu, H.M. (2000) Anticancer Activity Evaluation of the Solanum Glycoalkaloid Solamargine. Triggering Apoptosis in Human Hepatoma Cells. Biochemical Pharmacology, 60, 865-1873. https://doi.org/10.1016/S0006-2952(00)00506-2

[43] Liang, C.H., Liu, L.F., Shiu, L.Y., Huang, Y.S., Chang, L.C. and Kuo, K.W. (2004) Action of Solamargine on TNFs and Cisplatin-Resistant Human Lung Cancer Cells. Biochemical and Biophysical Research Communications, 322, 751-758. https://doi.org/10.1016/j.bbrc.2004.07.183

[44] Xie, X., Zhang, X., Chen, J., Tang, X., Wang, M., Zhang, L., Guo, Z. and Shen, W. (2019) $\mathrm{Fe}_{3} \mathrm{O}_{4}$ Solamargine Induces Apoptosis and Inhibits Metastasis of Pancreatic Cancer Cells. International Journal of Oncology, 54, 905-915. https://doi.org/10.3892/ijo.2018.4637

[45] Fu, R., Wang, X., Hu, Y., Du, H., Deng, B., Ao, S., Zhang, L., Sun, Z., Zhang, L., Lv, G. and Ji, J. (2019) Solamargine Inhibits Gastric Cancer Progression by Regulating the Expression of nc NEAT 1-2 via the MAPK Signaling Pathway. International Journal of Oncology, 54, 1545-1554. https://doi.org/10.3892/ijo.2019.4744

[46] Millward, M., Powell, A., Tyson, S., Daly, P., Ferguson, R. and Carter, S. (2005) Phase 1 Trial of Coramsine (SBP002) in Patient with Advanced Solid Tumors. Journal of Clinical Oncology, 23, 3105. https://doi.org/10.1200/jco.2005.23.16_suppl.3105

[47] Munari, C.C., de Oliveira, P.F., Campos, J.C.L., Martins, S.P.L., Da Costa, J.C., Bastos, J.K. and Tavares, D.C. (2014) Antiproliferative Activity of Solanum lycocarpum Alkaloidal Extract and Their Constituents, Solamargine and Solasonine, in Tumor Cell Lines. Journal of Natural Medicines, 68, 236-241. https://doi.org/10.1007/s11418-013-0757-0

[48] Tambuscio, A., Siliprandi, L., Dario, M., Cham, A., Cham, B. and Bordignon, M. (2016) Treatment of Cutaneous Carcinomas with a Topical Cream Containing Solasodine Rhamnosides: Focus on Efficacy, Compliance and Cosmetic Outcomes. European Academy of Dermatology and Venereology Congress, Athens, 19-22 May 2016, p. 0248.

[49] Cham, B.E. (2007) The Eggplant Cancer Cure: A Treatment for Skin Cancers and New Hope for Other Cancers from Nature's Pharmacy. Smart Publications.

[50] Cham, B.E. (2007) Solasodine Rhamnosyl Glycosides in a Cream Formulation Is Effective for Treating Large and Troublesome Skin Cancers. Research Journal of Biological Sciences, 2, 749-761. 
[51] Cham, B.E. (2009) When Does Alternative Become Orthodox? Skin Cancer Treatment with Solasodine Rhamnosyl Glycosides in Its Transitional Stage, a Case Study. Evidence-Based Complementary and Alternative Medicine, 6, 415-420.

[52] Cham, B.E. (2011) Topical Solasodine Rhamnosyl Glycosides Derived from the Eggplant Treats Large Skin Cancers: Two Case Reports. International Journal of Clinical Medicine, 2, 473-477. https://doi.org/10.4236/ijcm.2011.24080

[53] Chase T.R. (2011) Curaderm ${ }^{\mathrm{BEC} 5}$ for Skin Cancers, Is It? An Overview. Journal of Cancer Therapy, 2, 728-745. https://doi.org/10.4236/jct.2011.25099

[54] Cham, B.E. (2012) Intralesion and Curaderm ${ }^{\mathrm{BEC} 5}$ Topical Combination Therapies of Solasodine Rhamnosyl Glycosides Derived from the Eggplant or Devil's Apple Result in Rapid Removal of Large Skin Cancers. Methods of Treatment Compared. International Journal of Clinical Medicine, 3, 115-124.

https://doi.org/10.4236/ijcm.2012.32024

[55] Cham, B.E. (2013) Topical Curaderm ${ }^{\mathrm{BEC} 5}$ Therapy for Periocular Nonmelanoma Skin Cancer: A Review of Clinical Outcomes. International Journal of Clinical Medicine, 4, 233-238. https://doi.org/10.4236/ijcm.2013.45041

[56] Cham, B.E. (2013) Solasodine Glycosides: A Topical Therapy for Actinic Keratosis. A Single-Blind, Randomized, Placebo-Controlled, Parallel Group Study with Cura$\operatorname{derm}^{\text {BEC5 }}$. Journal of Cancer Therapy, 4, 588-596.

https://doi.org/10.4236/jct.2013.42076

[57] Cham, B.E. (2014) A Review of Solasodine Rhamnosides Therapy for In-Situ Squamous Cell Carcinoma on the Penis. British Journal of Medicine and Medical Research, 4, 621-631. https://doi.org/10.9734/BJMMR/2014/4677

[58] Cham, A. and Cham, B.E. (2015) Treatment of Skin Cancer with a Selective Apoptotic-Inducing Curaderm ${ }^{\mathrm{BEC} 5}$ Topical Cream Containing Solasodine Rhamnosides. International Journal of Clinical Medicine, 6, 326-333. https://doi.org/10.4236/ijcm.2015.65042

[59] Cham, B.E., Cham, K., Cham, A., Chase, T. and Zhou, V. (2015) Treatment of Non Melanoma Skin Cancers: An Intra-Comparison Study of Curaderm ${ }^{\mathrm{BEC} 5}$ and Various Established Modalities. Journal of Cancer Therapy, 6, 1045-1053. https://doi.org/10.4236/jct.2015.612114

[60] Punjabi, S., Cook, L.J., Kersey, P., Marks, R. and Cerio, R. (2008) Solasodine Glycoalkaloids: A Novel Topical Therapy for Basal Cell Carcinoma. A Double Blind, Randomized, Placebo-Controlled, Parallel Group, Multicentre Study. International Journal of Dermatology, 47, 78-82.

https://doi.org/10.1111/j.1365-4632.2007.03363.x

[61] Bordingnon, M. (2016) Efficacy and Safety of Curaderm ${ }^{\mathrm{BEC} 5}$ in the Treatment of Basal Cell Carcinoma: A Pilot Study in the Italian Population. European Academy of Dermatology and Venereology Congress, Athens, 19-22 May 2016, p. 0259.

[62] Dobrokhotova, F.Z., Betsev, A.F. and Belysheva, T.S. (2016) The Use of Kuraderm Drug in the Treatment of Basal Cell Carcinoma of the Head and Neck. Head and Neck Tumors, 6, 22-26. https://doi.org/10.17650/2222-1468-2016-6-3-22-26

[63] Xie, X., Zhu, H., Zang, J., Wang, M., Zhu, L., Guo, Z., Shen, W. and Wang, D. (2017) Solamargine Inhibits the Migration and Invasion of HepG 2 Cells by Blocking Epithelial-to-Mesenchymal Transition. Oncology Letters, 14, 447-452. https://doi.org/10.3892/ol.2017.6147

[64] Swetter, S.M., Tsao, H., Bichakjian, C.K., et al. (2019) Guidelines of Care for the Management of Primary Cutaneous Melanoma. JAAD, 80, 208-250.

https://doi.org/10.1016/j.jaad.2018.08.055 
[65] Chase, T., Cham, K.E. and Cham, B.E. (2020) Evaluation of Dermal Irritation and Skin Sensitization by Curaderm. International Journal of Clinical Medicine, 11, 548-558. https://doi.org/10.4236/ijcm.2020.119047

[66] Chase, T., Cham, K.E. and Cham, B.E. (2020) Curaderm the Long-Awaited Breakthrough for Basal Cell Carcinoma. Vol. 11. 